\title{
Diabetes y Embarazo
}

\author{
Dr. Gustavo Gómez Tabarez*
}

\section{DETECCION}

El enfoque del problema se orienta hacia:

1. La detección precoz de un estado diabético durante del embarazo que desaparece cuando la gestación termina (diabetes gestacional) o tipo II.

2. Hacer el diagnóstico de diabetes en una paciente de riesgo que no había sido detectado antes de embarazarse, que no se espera que desaparezca después del parto y que se empeora con el embarazo (diabetes química) o tipo II.

3. El manejo de la paciente diabética ya diagnosticada y en tratamiento que se ha embarazado (diabetes franca) o tipo I.

Todas las pacientes embarazadas que no se conozca como diabética pero que presente los siguientes factores de riesgo, deben ser objeto de estudio para descartar una diabetes durante el embarazo.

1. Antecedentes familiares de diabetes

2. Antecedentes de R.N. $>4.000$ grs. en embarazos anteriores.

3. Antecedentes de mortinatos

4. Antecedentes de malformaciones congénitas en productos de embarazos anteriores.

Universidad del Valle. Facultad de Salud. Departamento de Obstetricia y Ginecología.
5. Antecedentes de muertes neonatales tempranas o S.D.R. en recién nacidos.

6. Abortos a repetición.

7. Toxemia o prematurez en los embarazos anteriores.

8. Obesidad

9. Aumento excesivo de peso durante el embarazo actual.

10. Polihidramnios

11. Moniliasis vulvo-vaginal recurrente anterior o durante el embarazo actual.

En pacientes con embarazos mayores de 28 semanas se debe solicitar una CTOG y nunca dejar la investigación en una determinación de glicemia pre y postprandial únicamente.

En embarazos de menos de 28 semanas, con factores de riesgo y en vista de la importancia de hacer un diagnóstico precoz de diabetes durante el embarazo que cursa en la actualidad (40), se recomienda practicar una prueba con 50 grs. de glucosa. Se toma una glicemia pre-prandial y luego de 50 grs. de glucosa se determina una glicemia a la hora (1 hora).

Si esta última es de $\geqslant 140 \mathrm{mg} / \mathrm{dl}$ se considera que el paciente debe ser sometido a la CTOG, (3233). Si ésta es negativa, se debe repetir a las 28-30 semanas de gestación (Symposium on Gestational Diabetes) (1). La curva de tolerancia oral a la glucosa en una paciente de riesgo, negativa a las 28 semanas, debe repetirse a las $32-34$ semanas. 
Aunque The American Diabetic Association Workshop-Conference sobre Diabetes Gestacional (34) consideró que si bien hay otras pruebas de rastreo ("Screening") aceptables, el test de 50 grs propuesto originalmente por O'Sullivan $(35,36)$ ha sido suficientemente probado y efectivo con un $79 \%$ de sensibilidad y un $87 \%$ de especificidad. Beard (38) calculó que el costo de la detección con el test propuesto de 50 grs es de $40 \%$ menos que si se hace directamente la CTOG. Lavin (39) encontró que de los test de carga con 50 grs positivos ( $5.9 \%$ ), el $1.5 \%$ tuvieron una CTOG anormal.

\section{CTOG}

El tiempo más aconsejable para ordenar este examen es bien temprano en el tercer trimestre del embarazo ( 28 S.) si no existe razón para hacerlo antes (2-3-4-5-6).

La American Diabetics Association (7) recomienda estas instrucciones para practicar la CTOG y poder tener parámetros de comparación de acuerdo a los resultados obtenidos por los diferentes autores. Estos criterios han sido reafirmados por un reciente grupo de trabajo en Diabetes: (8).

a. Consumo libre de carbohidratos los 3 días anteriores (150-200 grs).

b. No consumir alcohol después de la comida del día anterior.

c. El test debe realizarse después de 8 horas de ayuno, pero no más de 16 . Sólo puede tomar agua según necesidad.

d. Idealmente la paciente debe estar ambulatoria durante los 3 días anteriores a la prueba.

e. 100 grs, de glucosa durante 5 minutos debe ser ingerido por el paciente y se contabiliza inmediatamente se comienza a ingerir la solución (23-24).

Algunos autores han usado 50 grs, de glucosa. Sin embargo, una dosis de 100 grs. es la más recomendable pues se ha comprobado que con esta carga de glucosa se detecta mejor un transtorno leve de metabolismo de carbohidratos en el tercer trimestre (25).

f. No se permite fumar, ni beber algo diferente a agua. g. Se toman muestras de sangre venosa antecubital exacto a una, dos y tres horas posterior a la ingestión.

\section{INTERPRETACION}

En vista de que no tenemos parámetros propios para interpretar la anormalidad de las respuestas, los estudios de O'Sullivan (9) realizados con este método en 752 pacientes durante el segundo y tercer trimestres del embarazo, calculando los resultados con dos variaciones standar y escogidos porque a 15 años el $60 \%$ de riesgo cumulativo de una diabetes establecida en esas pacientes. Los valores aceptados son:

\begin{tabular}{|lccc|}
\hline & Sangre venosa & Plasma venoso & Sangre capilar \\
\hline Ayunas & 90 & 105 & 90 \\
Una Hora & 165 & 190 & 170 \\
Dos Horas & 145 & 165 & 145 \\
Tres Horas & 125 & 145 & 125 \\
\hline
\end{tabular}

En el servicio usamos ahora un Dextrometer "Ames" que analiza muestras de sangre capilar para determinaciones urgentes. El método más corrientemente usado es en la actualidad el de la glucosaoxidasa, también realizado en el Servicio.

Si el valor en ayunas es $>90$ se clasifica la paciente como una Diabética química. Dos valores anormales diferentes del valor en ayunas clasifican la paciente como Diabética gestacional.

\section{CUADRO 1}

\section{DETECCION DE DIABETES EN PACIENTES \\ A RIESGO DURANTE EL EMBARAZO DETECCION B}

\begin{tabular}{|l}
$\begin{array}{l}\text { Antes } 28 \text { Semanas } \\
\text { Prueba de Carga } \\
\text { con } 50 \text { grs. } \\
\text { glucosa }(\mathrm{TCG})\end{array} \quad$ Ayunas $\leqslant 90 \mathrm{gr} \%$ \\
\hline
\end{tabular}

Si T. CG es positivo se recurre: CTOG con 100 grs glucosa Si T. CG es negativo se hará CTOG a las $28-30 \mathrm{~S}$.

\section{Manejc}

El tratamiento básico inicial es la dieta. Se instaura una dieta de $30-35 \mathrm{~K}$. cal. x Kg. de peso ideal, no siendo inferior a $1.600 \mathrm{cal}$. ni superior a $2.200 \mathrm{~K}$ cal. repartido en $50 \% \mathrm{CH}$., 30 grasas y 20 
proteínas repartidas en 2/7 al desayuno, 2/7 al almuerzo, $2 / 7$ a la comida y $1 / 7$ a tiempo de acostarse ( 8 a.m. -12 m. -5 p.m.-9p.m.). No se debe permitir un excesivo aumento de peso, pero un aumento de $10-11 \mathrm{~kg}$ puede ser ideal aún para la paciente no diabética (un incremento de $\pm 1.1 \mathrm{~kg}$. $\mathrm{x}$ mes. Una excesiva deprivación de ingesta calórica puede llevar fácilmente a cetosis a la paciente diabética embarazada, especialmente durante el ayuno o la deprivación calórica (14). En algunas pacientes diabéticas gestacionales severas, o sea aquellas en que no se pueden controlar con dieta, debe recurrirse a microdosis de insulina de duración intermedia. En estos casos debe iniciarse con 10 UI NPH (microdosis) y se incrementará hasta 15 UI, en una sola dosis matinal a las 8 a.m. concomitantemente con el desayuno. Se considera bien controlada una paciente diabética gestacional cuando la glicemia en ayunas es $\leqslant 90 \mathrm{mgrs} / \mathrm{dl}$ y la glicemia post prandial de 2 horas es $\leqslant 140 \mathrm{mg} / \mathrm{dl}$. El manejo de la diabética gestacional es ambulatorio a no ser que se presente una complicación que requiera su hospitalización. Se controla en la Clínica de Embarazo de Alto Riesgo, 2 veces por mes, con glicemias pre y post prandiales que se anotan en la hoja de control ambulatorio de pacientes diabéticas, diseñada para tal efecto. El manejo obstétrico y la monitoría biofísica y bioquímica sigue los mismos parámetros que más adelante especificaremos para la paciente insulinodependiente y dependiendo también de la evolución del embarazo.

\section{Insulino - Dependientes}

Debe hospitalizarse cuando se haga el diagnóstico de embarazo para determinar los requerimientos de insulina y ajustar la dieta. Para la administración de insulina hay que tener en cuenta los siguientes cambios metabólicos durante el embarazo:

a. Los requerimientos de insulina en el primer trimestre tienden a ser menores al parecer por un aumento de la sensibilidad a insulina. Aún la CTOG se mejora en esta etapa del embarazo debido a la disminución del efecto de las hormonas gastrointestinales en la secreción de insulina (26) y la utilización de la glucosa materna por el feto.

b. Los niveles de glicemia en ayunas en la paciente embarazada son significativamente menores desde temprano en la gestación y ésta disminuye a medida que el embarazo progresa debido a la contribución de la glucosa materna al sistema metabólico fetal, el cual requiere de aquella como combustible energético (6). Du- rante las horas del sueño nocturno estos niveles son aún más bajos por la falta de ingesta de la madre.

c. A medida que el embarazo progresa aumentan los requerimientos de insulina, que pueden llegar hasta 75 ó $100 \%$ de las utilizadas pre embarazo. La responsabilidad mayor de que ésto suceda es la producción ascendente de hormonas placentarias "anti-insulínicas", principalmente HPL.

d. La hipoglicemia materna, a la cual se agrega hipoalaninemia por trasferencia pasiva al feto, hacen que el ayuno en la embarazada y en la diabética embarazada, con excesiva tendencia a la hipoglicemia, incrementa la producción de ácidos grasos libres, cuerpo cetónico y glicerol.

e. Tanto la hiperglicemia como la hipoglicemia son responsables de la tendencia a formación de cuerpos cetónicos en la diabética embarazada. Ambas pueden ocurrir con mayor frecuencia en el tercer trimestre. La excesiva utilización de insulina o una dieta muy severa en reducción de agentes calóricos externos (carbohidratos) (8) hacen que se incremente HPL, lo cual resulta en aumento de lipolisis. La hiperglicemia actúa incrementando la acción antinsulínica, lo mismo que los niveles de HPL, con los efectos descritos anteriormente.

f. La hipoglicemia nocturna (con la posible cetosis asociada) es un peligro real, especialmente en el tercer trimestre del embarazo y principalmente en la diabetes tipo I $(16,29)$. La "difusión facilitada" de glucosa materna durante la noche, al feto, la falta de ingesta de la madre y la utilización de insulinas en dosis vespo-nocturnas, contribuyen al fenómeno.

Se inicia el manejo con una dósis única de insulina NPH. De acuerdo con los valores de glicemia en ayunas siguiendo este esquema:

$>100$ hasta $120 \mathrm{mg} / \mathrm{dl}: 10 \mathrm{UI}$;

120 - 150: 15 UI

$150-180: 20 \mathrm{UI}$

Esta dosis de ingreso se ajustará cada $48 \mathrm{~h}$. durante su permanencia en el Servicio.

NOTA: La dosis de Insulina NPH a las 8:00 am., tiene su efecto máximo durante la hiperglice- 
mia postprandial del almuerzo (3 pm.) y previene la hiperglicemia previa a la comida (5 p.m.) con efecto que dura hasta la mañana siguiente (18) (37). Esta acción junto con la comprobada hipoglicemia nocturna de la mujer embarazada explica el por qué algunas pacientes se pueden controlar con este simple esquema, especialmente cuando el requerimiento de insulina es bajo y por lo tanto la diabetes es aún moderada (16).

CUADRO No. 2

\section{DIAGRAMA DE MANEJO PACIENTE INSULINO DEPENDIENTE CON UNA SOLA DOSIS DE INSULINA NPH Y DIETA EN SEPTIMOS}

\begin{tabular}{|cccc|}
\hline Hora & NPH & Glicemia & Dieta \\
\hline 7:00 am. & \multirow{3}{*}{ Glicemia en Ayunas } \\
8:00 am & $\mathrm{X}$ & & Desayuno 2/7 \\
$12: 00 \mathrm{~m}$. & & Glicemia & Almuerzo 2/7 \\
$3: 00 \mathrm{pm}$. & & & Comida 2/7 \\
$5: 00 \mathrm{pm}$. & Glicemia & Colación $1 / 7$ \\
$8: 00 \mathrm{pm}$. & & & \\
$9: 00 \mathrm{pm}$ & & & \\
\hline
\end{tabular}

Sobre este esquema inicial que debe observarse por lo menos $48 \mathrm{hrs}$. antes de cambiar el esquema de insulina o dieta se ajustan y determinan los posteriores esquemas terapéuticos.

Si la glicemia en ayunas persiste $>90 \mathrm{mg} / \mathrm{dl}$ pero la glicemia $3 \mathrm{pm}$. es $<140 \mathrm{mg} / \mathrm{dl}$ se agregará una dosis de insulina NPH aplicada a las 5pm. que se puede iniciar con 5UI si el valor en ayunas no es mayor de 100 , de $10 \mathrm{UI}$ si el valor es $>110 \mathrm{mg} \%$ y así sucesivamente, valorando su acción durante 48 horas, antes de cambiar el régimen de insulina instaurado (Cuadros 4 y 5 ).

Si la glicemia de las 3 p.m. persiste alta se aumentará la dosis de las 8 a.m. siempre y cuando no se observe hipoglicemia nocturna. En este último caso generalmente se controla con cambios en la dieta respecto a hora y cantidad administrada. Hasta ahora no hemos necesitado emplear insulina cristalina adicional.

La utilización de la mezcla NPH - Crist. en dosis nocturna es inconveniente y peligrosa por la posibilidad de hipoglicemia que lleva la paciente a cetosis irremediablemente (7).

Una vez logrado el control metabólico de la paciente se controla por C.E. en E.A.R., cada 2 se- manas con glicemia en ayunas y postprandial $3 \mathrm{pm}$. Se ha elaborado una hoja que la paciente ambulatoria guarda y en la que se registran los valores de glicemia, la dosis de insulina recibida, la dieta recibida y donde la paciente hace sus observaciones acerca de eventos especiales como hipoglicemia, movimientos fetales, y procedimientos especiales realizados.

Si el control ambulatorio de glicemia no es posible, debe hospitalizarse de nuevo la paciente cuatro semanas antes de la fecha probable de parto.

La dieta utilizada en estas pacientes seguirá regida por la administración de $30-35 \mathrm{Kcal} \mathrm{Kg} /$ peso ideal, repartida en $50 \% \mathrm{CH}, 30$ grasas y 20 proteínas y fraccionada en $2 / 7,2 / 7,2 / 7$, y $1 / 7$ sin ser inferior a 1.600 cal. o superior a 2.200 en pacientes obesas y delgadas respectivamente.

\section{FIGURA 1}

\section{ACCION DE INSULINA CRISTALINA (- - - ) E INSULINA NPH ( - ) EN ESQUEMA DE DOSIS UNICA O DOBLE}

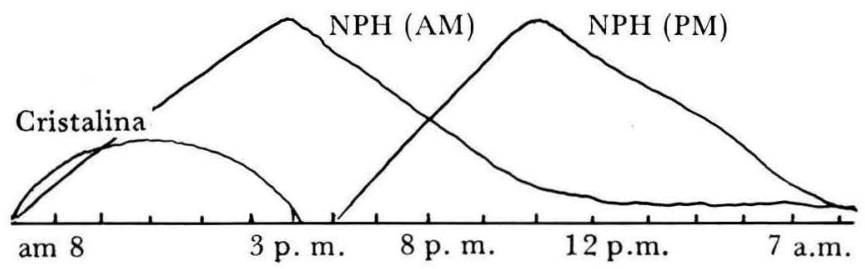

\section{Monitoría de la Diabética Embarazada}

Después de lograr mantener euglicémica la paciente $(80-90 \mathrm{mg} / \mathrm{dl}$ en ayunas) lo que evidentemente reduce la morbimortalidad fetal (40), el avance más importante en el manejo de estas pacientes es la aparición de métodos para monitorizar la madurez pulmonar fetal y detectar el bienestar fetal intrauterino. El doble fin de evitar la prematurez, a veces iatrogénica en el R.N. y la mayor incidencia de mortalidad intrauterina anteparto, se logra con la monitoría adecuada.

\section{Monitoría Bioquímica}

\section{Estriol Plasmático}

Cuando se cuenta con ensayos tan frecuentes como se quieran y se dispone de los resultados rápidamente es útil su medición. Estriol no conjugado parece ser el de más utilidad por su bajo porcentaje de falsos positivos (10\%) (16). 
Determinaciones semanales de la semana $30-$ 34 , y diaria desde la semana 34 en diabetes sin lesión vascular o retinopatía y desde la semana 32 en tipos de diabetes más avanzada es recomendada por Gabbe y Quilligan. Esta monitoría debe complementarse con la monitoría biofísica para disminuir los riesgos de falsos positivos que llevarán al obstetra a terminar prematuramente el embarazo.

\section{Hemoglobina AlC (Glicohemoglobina)}

La glicohemoglobina o $\mathrm{Hb} \mathrm{A} 1 \mathrm{C}$ es una variante normal de la hemoglobina humana A y se distingue de ésta porque tiene glucosa en enlace covalente con el $\mathrm{N}$ terminal de la cadena $\mathrm{B}$.

La $\mathrm{Hb}$ A $1 \mathrm{C}$ corresponde al $5 \%$ del total de hemoglobina en pacientes no diabéticos pero se incrementa 2 ó 3 veces en pacientes con intolerancia a la glucosa.

Diabéticas embarazadas presentan valores de $\mathrm{Hb}$ A1C significativamente más bajo que diabéticas no embarazadas (38). Pero hay muy buena correlación entre $\mathrm{Hb} \mathrm{A} 1 \mathrm{C}$, niveles de glicemia y peso del RN (39), lo que sugiere que la determinación de $\mathrm{Hb} \mathrm{A} 1 \mathrm{C}$ podría convertirse en una ayuda para el control de la paciente diabética embarazada. Sus niveles dan una información confiable sobre lo adecuado del control de glucosa en las semanas o meses previos. Ylinen encontró relación significante entre hipoglicemia e hiperbilirrubinemia fetal y aumento de niveles de $\mathrm{Hb} \mathrm{AlC}$ en la madre.

Se recomienda para el efecto de controlar lo adecuado del tratamiento durante las 4 semanas anteriores, una determinación mensual de $\mathrm{Hb} \mathrm{A} 1 \mathrm{C}$; si el control de la glicemia ha sido excelente, su glicohemoglobina no debe exceder del $6 \%$ de la hemoglobina total.

\section{Monitoría Biofísica}

\section{Prueba de No Stress}

Técnicamente fácil de realizar e interpretar debe iniciarse a partir de la semana 34 de embarazo. En pacientes con diabetes severa a las 32 semanas. Se repite cada 8 días en pacientes bien controladas desde el punto de vista metabólico y manejadas ambulatoriamente. En pacientes de difícil control y hospitalizadas, debe practicarse tan frecuente como sea necesario. Cada 5 días es el intervalo mayor entre cada monitoría y puede llegar a ser interdiaria o diaria (20-19).

\section{Ecografía}

Es útil donde se cuenta con el recurso para determinar crecimiento fetal (macrosomía y polihidramnios).

La determinación de la relación cabeza-tórax es importante para decidir la vía de terminación del embarazo. El perímetro abdominal es un buen parámetro ecográfico para determinar peso fetal (41). Un examen ecográfico inicial a las 20-22 semanas y posterior a la semana 32 y luego cada 4 semanas (21) dá magnífica información acerca de crecimiento fetal intrauterino, líquido amniótico, madurez placentaria y bienestar fetal (35).

\section{Movimientos fetales}

Desde el punto de vista clínico, la observación y cuantificación por la madre de los movimientos fetales en un período de 12 horas es un parámetro importante como indicador de monitoría más precoz y más estricta en estas pacientes.

Menos de 10 movimientos en 10 horas deben alertarnos. Se puede comenzar a instruir a la madre en esta práctica desde la semana 32 de embarazo (22).

CUADRO No. 3

\section{DIABETES Y EMBARAZO - MONITORIA BIOFISICA}

\begin{tabular}{|ccll|}
\hline $\begin{array}{c}\text { Semanas } \\
\text { Embarazo }\end{array}$ & $\begin{array}{l}\text { Tipo de } \\
\text { Diabetes }\end{array}$ & Frecuencia* \\
\hline 34 S. & PNS & Gestación. B-C (II) & 1 Semanal* \\
32 & PNS & Tipo I (DFR) & 2 Semanal* \\
$22-34$ & Ecografía & Todo Tipo & C/4 Semanas \\
30 & Movimientos & Todo Tipo & Diaria \\
& Fetales & & \\
\hline & La frecuencia se acorta de acuerdo con el resultado o \\
evolución clínica de la paciente, hasta una diaria.
\end{tabular}

Manejo Ante - Intra - Post Parto Inmediato. Tiempo y Vía de Evacuación.

El manejo peri-parto tiene características especiales y tiene que alcanzar diferentes metas:

a. Evitar la hipoglicemia materna post parto inmediato debido principalmente a la súbita caída de los niveles de HPL. 
b. La falta de ingesta durante este tiempo,agrava la hipoglicemia materna y por lo tanto induce la mayor producción de cuerpos cetónicos y ésto incrementa el riesgo SDR por baja de $\mathrm{pH}$ fetal $(30,31)$.

c. Por otra parte, la hiperglicemia en una madre mal controlada induce a hiperinsulinemia fetal que es la responsable de la hipoglicemia neonatal a veces tan severa que lleva el niño a convulsionar.

\section{Inducción - Cesárea}

Manejo: Se utiliza 1/2 dosis de la insulina utilizada antes del embarazo (32), la mañana que se inicia la inducción.

Una solución de dextrosa $5 \%$ a una dosis de no más de $100-200 \mathrm{ml}$. por hora (5-10 grs.), minimiza la hiperglicemia materna y por lo tanto la hipoglicemia fetal (33). Después de parto dosis suplementarias de insulina cristalina si el test de orina en glucosa muestra $(3+$ ó $4+$ ).

El día siguiente al parto existe un relativo incremento de sensibilidad a la insulina, puesto que las hormonas diabetogénicas placentarias son rápidamente metabolizadas y sacadas de circulación. Se utiliza 1/3 de la dosis de insulina utilizada previa al embarazo y el ajuste diario se hace de acuerdo con los valores de glicemia en día subsiguientes y usualmente alrededor del 7 o. día post-parto. Hay pacientes que persisten varios meses con su aumento de sensibilidad a la insulina en la época post-parto.

Si la paciente va a ser evacuada por cesárea, la dosis de insulina se aplicará inmediatamente después del procedimiento quirúrgico. El tiempo de evacuación estará dado por la madurez pulmonar fetal, representada por la relación $\mathrm{L} / \mathrm{S}$ y/o Test de Clement y por el estado fetal intrauterino. La mayor incidencia de muertes fetales intrauterinas a medida que avanza el embarazo es debida, entre otras causas, a la disminución del flujo útero placentario y la exposición del feto a acidosis intrauterina en pacientes inadecuadamente manejadas desde el punto de vista metabólico.

Si la Monitoría Biofísica (Prueba de No Stress y/o Prueba de Tolerancia o la Oxitocina) indica bienestar fetal y la amenorrea es confiable, se realizará una amniocentesis a las 38 semanas de gestación en pacientes diabéticas Tipo I y a las $40 \mathrm{~S}$., en dia'béticas Tipo II, para determinar Test de Clement y relación $\mathrm{L} / \mathrm{S}$. Si la relación $\mathrm{L} / \mathrm{S}$ en 2.5 o más y el
Clement $(+)$, se evacúa. Lo ideal es determinar fosfatidilglicerol, que nos proporciona un dato más confiable acerca de madurez pulmonar en la diabética. En pacientes en que no hay amenorrea confiable, a pesar de tener determinación y valoración ecográficas de edad gestacional, debe practicarse la amniocentesis para determinar el estado pulmonar del feto.

Si existe indicación obstétrica, la evacuación será por vía alta. En fetos macrosómicos determinados clínicamente y confirmados con Ecografía debe ser por cesárea. Fetos mayores o iguales de 4.000 grs., además de representar una distocia obstétrica, son mucho más susceptibles a acidosis durante el trabajo de parto y especialmente en el expulsivo (40). Otras indicaciones de cesárea son:

a. Cetoacidosis, pielonefritis clínica y toxemia sobreagregada (signos de mal pronóstico de Pederson) (34).

b. Primer embarazo en una paciente mayor de 30 años.

c. Antecedentes de mortinatos repetidos y

d. Abortadora habitual

Cuando la determinación es parto vaginal, éste debe ser monitorizado continuamente.

\section{DIABETES Y EMBARAZO}

\section{Manejo Intraparto - Postparto}

- Determinar Madurez Pulmonar Fetal (L/S Clement).

- Utilización de $1 / 2$ dosis de Insulina pre-embarazo el día Inducción.

- Infusión de Dextrosa 5 \% AD Gr./H. (100 ml) en pacientes que llegan bien controladas al parto.

- Si se decide una Operación Cesárea, la dosis de Insulina NPH se aplica inmediatamente después del acto quirúrgico.

- De acuerdo con la Glicemia, se usará $1 / 2$ ó $1 / 3$ de Dosis NPH Pre-embarazo durante 5-7 días (Postparto). 


\section{Manejo de Cetoacidosis}

- Monitoría C/2 - 4H. de glicemia - Bicarbonato - Potasio

- 25 UI Insulina cristalina E.V.

- $\quad 10-20$ UI Insulina cristalina cada hora

- $\quad 1-3$ litros de solución salina en 6-12 HS, de acuerdo con estado de hidratación.

$100 \mathrm{~m} / \mathrm{Eq}$ en primer litro de líquido SI pH 7.25 ó $10 \mathrm{~m} \mathrm{Eq/L} \mathrm{de} \mathrm{bicarbonato.}$

$10 \mathrm{~m} / \mathrm{Eq}, \mathrm{DE} \mathrm{K} \mathrm{EV} / \mathrm{H}$ a partir de la cuarta hora de infusión.

Control estricto de diuresis.
CUADRO No. 4 DIABETES Y EMBARAZO - ESQUEMA DE MANEJO
DOSIS UNICA

\begin{tabular}{|c|c|c|c|c|}
\hline Fecha & Hora & Glicemia & Dieta & Insulina \\
\hline & $\begin{array}{c}\text { 7:00 am. } \\
\text { 8:00 am. } \\
12: 00 \mathrm{~m} . \\
3: 00 \mathrm{pm} . \\
5: 00 \mathrm{pm} . \\
8: 00 \mathrm{pm} . \\
9: 00 \mathrm{pm} .\end{array}$ & $\begin{array}{l}x \\
x\end{array}$ & $\begin{array}{l}\mathrm{D} \times 2 / 7 \\
\mathrm{~A} \times 2 / 7 \\
\mathrm{C} \times 2 / 7 \\
\mathrm{Ca} \times 1 / 7\end{array}$ & $\begin{array}{l}\mathrm{NPH} 3 / 3 \\
{ }^{\circ} \mathrm{MANPH}\end{array}$ \\
\hline
\end{tabular}

CONTROL DIARIO DE LA PACIENTE DIABETICA

\begin{tabular}{|c|c|c|c|c|c|c|}
\hline Fecha & Hora & Glicemia & $\begin{array}{c}\text { Insulina } \\
\text { N.P.H. }\end{array}$ & Insulina & Cetonuria & $\begin{array}{c}\text { Hora } \\
\text { Alimentación }\end{array}$ \\
\hline & & & & & & \\
& & & & & & \\
\hline
\end{tabular}

\section{BIBLIOGRAFIA}

1. Symposium on Gestational Diabetes. Diabetes Care 3:399, 1980

2. CAMPBEll, PYKe, TAYloR, J. Obs. Gynecol. Br, Commonw: 78:498, 1971.

3. O'SUlLIVAN; et al. J. Clin. Endocrinol, Metab. 3: 33, 1970.

4. TYSON; Am. J. Obst. Gynecol. 125:1009, 1976.

5. O'SULLIVAN. Diabetes. 313:18, 1963

6. MERKATZ et al. Diabetes Care. 3: 453, 1980.

7. Committee on Statistic of American Diabetes Association: Diabetes 18: 299, 1969.

8. Symposium on Gestational Diabetes. Diabetes Care. 3: 399; 1980.

9. O' SUlLiVAN. AM. J. Obst. Gynecol. 116:895, 1973.

10. Maternal Diabetes Data group. Diabetes 28: 1039, 1979.

11. KNOPP O' SULLIVAN. Endocrinology: 92: 984, 1973.

12. YEN. Gynecol. 136: 483, 1980 ,

13. HARE and WHITE. Diabetes 3: 394, 1980.

14. FELIG. P. American J. Clin. Nutr. 26: 998, 1973.

15. SEED, E. Clin. Obst. Gynecol. 24: 1: 57.

16. GILLMER BR. Med. J. 3: 309, 1975.

17. JOVANOVIC. Am. J. Med. 68: 105, 1980

18. SEED E. Clin. Obstet. Gynecol. 24: 1: 59.

19. GGEBELSMAN. Am. J. Obst. Gynecol. 130: 424, 1978.

20. GOE BELSMAN, Am. J. Obst. Gynecol. 135: 764, 1979.

21. HOBBINS. Am. J. Obst. Gynecol. 128: 881, 1977.

22. PEARSON. Br. Med. J. 1: 1305, 1976.
23. LINDE BR. Commonw. 80: 1033, 1973.

24. O'SULLIVAN. Gestational Diabetes and its significance: Early Diabetes. New York. Academic Press., 1980.

25. KNOPP. Clin. Obst. Gynecol. 24: 1: 32.

26. HORNNES J. Clin. Endocrinol. Metab. 48: 506, 1979.

27. FRENKEL. On The Nature and Treatment of Diabetes. Excepta Medica Foundation, 1965.

28. MINTZ. Diabetes Mellitus and Pregnancy Diabetes Care. 1:4, 9, 1978.

29. YEN. Reproductive Endocrinology. Philadelphia. Saunder, 1978.

30. HOEBEL G.J. Obstet. Gynecol. 39:82, 1972.

31. SEEDS A.E. Am. J. Obstet. Gynecol. 135:887, 1979.

32. WHITE P. Med. Clin. North Am. 49: 1015, 1965.

33. LIGTH. Am. J. Obstet. Gynecol. 113:345, 1972.

34. PEDERSON. La Diabética gestante y su recién nacido. Salvat Editores, 1971.

35. O'SUllivan J.; MAHAN C.; CHARles D. Am. J. Obstet. Gynecol. 116:895, 1973.

36. O'SULLIVAN J.; MAHAN. Diabetes Care. 3:437, 1980.

37 O'SULLIVAN J., MAHAN C: Diabetes 13,278, 1964.

38. BEARD R.; GILLNER G. G.; OAKLEY N. Diabetic Care 3:468, 1980.

39. LAWIN J.R.; et al. Am J. Obst. Gynecol. 141:491, 1981.

40. JOVANOVIC, DRUZIN, PETERSON, AM. J. Med. 71:921, 1981.

41. FESCINA. I Curso Ecografía en Medicina Perinatal, 1983. Cali. 